\title{
A Comparative Analysis between Low Loss Kagome Structured THz Hollow Core and Porous Core PCF
}

\author{
Mohaiminul Islam, Md. Anwar Hossain, and Fahmida Haque
}

\begin{abstract}
This paper presents a comparative study between a porous core kagome lattice photonic crystal fiber (PCF) and a air filled Hollow core kagome lattice PCF (HC-PCF), which acts as a low loss and polarization maintaining tera hertz (THz) waveguide. Proposed PCFs are simulated using finite element method (FEM), including the effective material loss (EML), confinement loss and single mode propagation. Cyclic olefin copolymer, also known as TOPAS material have been chosen for the air micro structured inhibited coupled design of PCF which has the lowest bulk material absorption loss of $0.2 \mathrm{~cm}^{-1}$. Expressions are given to asses this optimization and the result are shown for the variation of core diameter from $425 \mu \mathrm{m}$ to $600 \mu \mathrm{m}$ for both the Hollow core and porous core PCFs and for the comparison with porous core the optimized porosity taken as $60 \%$ and $70 \%$ along with the frequency of $1 \mathrm{THz}$. The analysis results approximately $80 \%$ to $90 \%$ improvement in losses in case of passing THz waves through HC-PCF rather than Porous core PCF. By varying core parameter such as core porosity and core diameter and strut width at $1 \mathrm{THz}$, the goal is to show an optimized solution consisting of low EML and confinement loss for both of the wave guidance and comparing the results to analyze and hence finding out a better solution.
\end{abstract}

Keywords-THz Waveguide; kagome; EML; confinement loss;

\section{INTRODUCTION}

Terahertz (THz) waves are the non-ionizing electromagnetic waves within the International Telecommunication Union (ITU) designated band of frequencies from 0.1 to $10 \mathrm{THz}$ [1] which lies between the electronic and optical region in the electromagnetic wave spectrum, known as $\mathrm{THz}$ gap. The terahertz is used as an indicator of the frequency of infrared (IR), visible, and ultraviolet (UV) radiation [2]. The properties and behavior of $\mathrm{THz}$ waves are governed by the Maxwell equations, just like

\footnotetext{
Mohaiminul Islam

Graduate Student, Dept. of EEE

American International University-Bangladesh (AIUB).

Dhaka, Bangladesh

e-mail: muhaiminul.mahe@ymail.com;

Dr. Md. Anwar Hossain

Associate Professor; Dept. of EEE

American International University-Bangladesh (AIUB).

Dhaka, Bangladesh

e-mail: anwar.eee113@aiub.edu;
}

Fahmida Haque

Graduate, Dept. of EEE

American International University-Bangladesh (AIUB).

Dhaka, Bangladesh

e-mail: fahmida32@yahoo.com; the other waves for being an electromagnetic wave. $\mathrm{THz}$ spectrum has recently been attracting the attention of the researchers due to its drug testing [3], sensing, security, communications [4-5] and medical diagnostics [6-7].

In the beginning, due to the position of THZ in the electromagnetic spectrum between the microwave and infrared wave region, such waves were called far-infrared waves or millimeter/submillimeter waves. After the successful generation and detection of $\mathrm{THz}$ pulses by photoconductive switches in 1980s, early researchers were focused on developing technologies in generating, detecting, and manipulating $\mathrm{THz}$ waves [8]. Due to its intense popularity in various applications, the last decade has been flavored commercial availability of $\mathrm{THz}$ sources and detectors [9-10] but have scarcity of an efficient low-loss waveguide for $\mathrm{THz}$ wave transmission [11]. Low loss and commercially viable waveguides are essential for the successful implementation of commercially viable waveguide. Thus, for the commercial implementation of low loss waveguide researchers are facing challenges to design confined high power fraction in dry air and low power fraction within materials [12-13].

Photonic crystal fibers (PCFs) are optical fibers, based on the properties of photonic crystal that have wavelength-scale morphological microstructure running along their length [14] PCFs offer the opportunity to fabricate optical waveguide with enhanced linear and nonlinear optical properties [15] in a more efficient way overcoming the limitation of the conventional fiber optics. PCFs are proving to have a multitude of important technological and scientific applications. The main dielectric waveguides for $\mathrm{THz}$ guidance specially focused on hollowcore, solid-core, and porous-core waveguides with different wave guiding mechanism. Many researchers are investigating the low-loss and low dispersion profile of porous core and hollow core PCFs for $\mathrm{THz}$ propagation, as solid core waveguides are losing researcher's interest due to its high absorption loss[16]. An effective environment for nonlinear optics provided by the hollow core PCF (HC-PCF) which helps to avoid beam diffraction by offering long interaction lengths [17] and broadband transmission can be achieved with moderately low loss. Hollow core waveguides use photonic band gaps or anti-resonances with immediate cladding structure for wave guidance and the porous core use total internal reflection as guiding mechanism [16].

The first Kagomé-lattice hollow-core microstructured fiber for $\mathrm{THz}$ wave guidance was proposed by $\mathrm{Lu}$ et al [18]. 
Research on reducing material absorption in Terahertz (THz) waveguides have been conducted by many researchers, which leads to the development of dielectric-lined hollow cylindrical metallic waveguides with transmission losses as low as 1 $\mathrm{dB} / \mathrm{m}$ [19]. Heckl et al. [20] have proposed a gas filled Kagome-type Hollow-Core Photonic Crystal Fiber which guided 30-fs pulses with pulse energy of more than $10 \mu \mathrm{J}$, which is more than five times higher than for any other photonic crystal fiber [21]. Another research has showed that effective Kerr nonlinearity of hollow-core kagomé-style photonic crystal fiber (PCF) filled with argon gas increases over 100 times when the pressure is increased from 1 to 150 bar, reaching $15 \%$ of that of bulk silica glass, while the zero dispersion wavelength shifts from 300 to $900 \mathrm{~nm}$ by Azhar et al. [22].The general features of the loss spectrum demonstrated by P. St. J. Russel with a simple model of kagome structure hollow-core PCF consisting of thin concentric hexagons of glass in air by numerical simulation and suggest ways to shift the high-loss resonances into the loss spectrum [23].

The first proposed novel honeycomb porous core photonic band gap fiber (PC-PBGF) by Nielsen et al. [13] was reported to show lowest effective material loss (EML) than the conventional PBGF. Liang et al. [24] has proposed a porous core photonic band gap fiber for guiding $\mathrm{THz}$ wave in a frequency range from $0.98 \mathrm{THz}$ to $1.15 \mathrm{THz}$ with a low loss, which they proposed can also provide a broader bandwidth and a much more flattened dispersion profile. An air filled porous core PCF for low loss guidance of $\mathrm{THz}$ have approached Uthma et al [25], where they approached a range of techniques including stack-and-draw, extrusion, and drilling for the fabrication of this proposed PCF. Numerical and experimental investigation has been showed for a low loss porous-core honeycomb fiber for terahertz wave guiding by Bao et al. [12] where they introduction of a porous core with hole size of the same dimension as the holes in the surrounding honeycomb cladding results in a fiber that can be drawn with much higher precision and reproducibility than a corresponding air-core fiber.

These papers has analyzed a kagome lattice PCF design structure [11] and also design the core as air-filled hollow core in order to show a comparative analysis to find out the lowest effective material loss with greater confinement from these types of $\mathrm{THz}$ wave guiding mechanism.

\section{Design STRUCTURe}

Theoretical and practical research is going on now-a-days on the porous core PCF's and the hollow core PCF's. Hollow core dielectric waveguides has low effective material loss (EML) but still THz transmission is limited to a selected range of frequency band [16]. At present research on $\mathrm{THz}$ frequency been going towards porous core because of its low EML and high mode power confinement in dry air

In this paper, design of a kagome lattice PCFs [11] has been analyzed and the core is further designed with the hollow core and a comparative analysis has been made to decide for the successful and well confined $\mathrm{THz}$ wave guidance PCF. The main reason of designing the core is to show a comparative analysis to determine the lowest material absorption loss between these two waveguides.

The proposed porous core kagome PCF is made with a hexagonal lattice of air holes consisting of 4 rings and 61 air holes including the centre air-hole. These hexagonal lattices are formed nicely inside the core with kagome structure. The cladding region is formed by large air holes with TOPAS material around the air-holes.

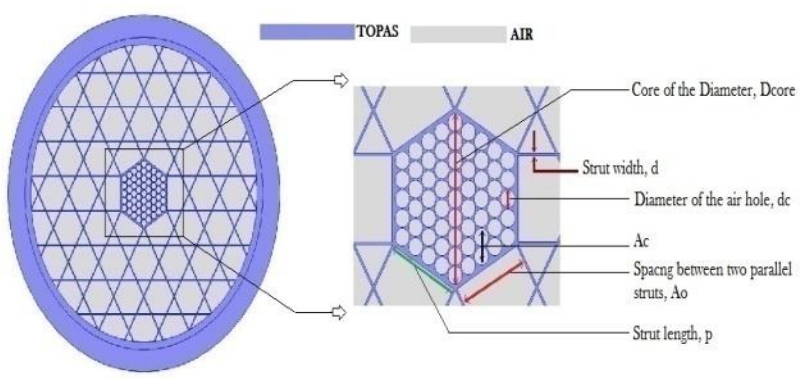

Fig. 1. Cross section of the analyzed design of the porous core kagome lattice PCF [11]

The geometry of the proposed kagome lattice PCF has been shown in fig.1 [11], where air holes are surrounded by TOPAS material. $\mathrm{D}_{\text {core }}$ is labeled as the diameter of the core which is actually the total diameter of the two opposite vertexes of the core inside. Its side length is labeled as $\mathrm{p}$. TOPAS is used as a background material because of its lowest bulk material absorption loss of $\alpha_{\text {mat }}=0.2 \mathrm{~cm}^{-1}[26]$. The refractive index is constant and value is, $n=1.5258$ [24]

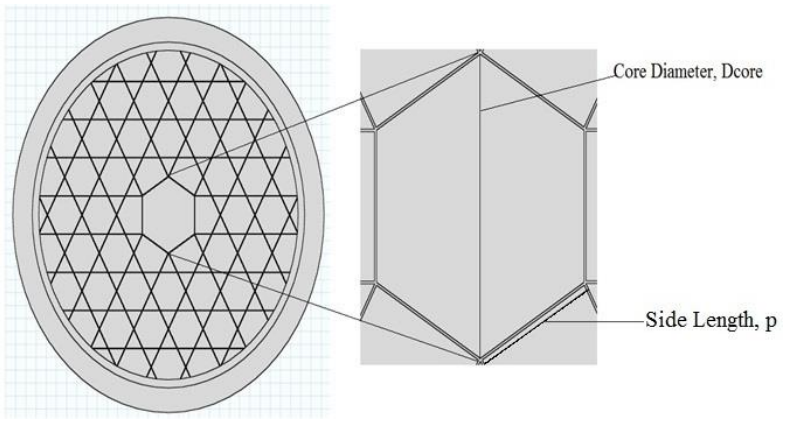

Fig. 2. Cross section of the analyzed design of kagome lattice HC-PCF

The proposed design of hollow core kagome lattice $\mathrm{THz}$ wave guide has been shown in fig. 2 . The core inside has been designed as a hexagonal air-filled hollow-core. In this design the air was pumped at 1000 bar to find the lowest EML possible. For both of the design others parameter such as: strut width, d; spacing between two parallel struts, Ao; side length, $\mathrm{p}$ remains same as the design structure proposed in [11].

The mode field confinement with $60 \%$ and $70 \%$ core porosity at $1 \mathrm{THz}$ frequency and $5 \mu \mathrm{m}$ strut width of the porous core PCF has been shown in the figure 3. From the figure it is observed that the mode field is very well confined at the core and have better confinement at $70 \%$ porosity.

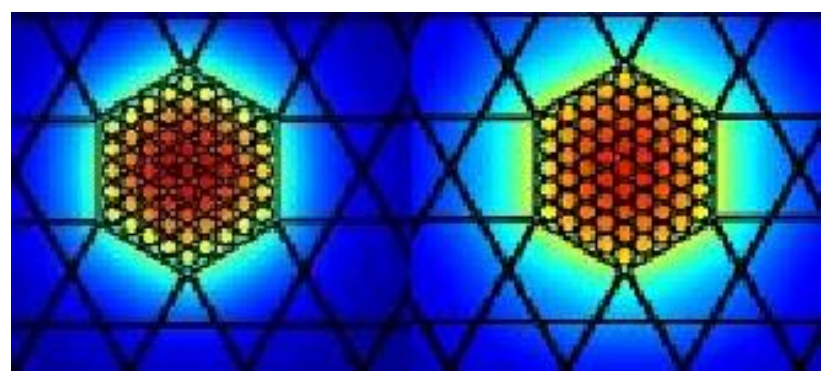

(a)

(b)

Fig. 3. Mode field confinement for porous core PCF for $1 \mathrm{THz}$ at (a) $60 \%$, (b) $70 \%$ core porosity and $\mathrm{d}=6 \mu \mathrm{m}$ 


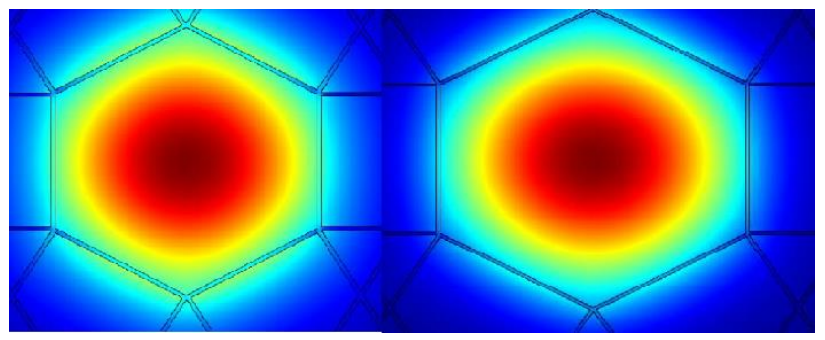

(a)

(b)

Fig. 4. Mode field confinement for $\mathrm{HC}-\mathrm{PCF}$ at $\mathrm{f}=1 \mathrm{THz}$ with (a) $425 \mu \mathrm{m}$ and (b) $600 \mu \mathrm{m}$ core diameter and at $\mathrm{d}=6 \mu \mathrm{m}$.

Fig.4 shows the mode field confinement of the HC-PCF at $1 \mathrm{THz}$ frequency for two different core diameter of $425 \mu \mathrm{m}$ and $600 \mu \mathrm{m}$ and fixed strut width at $6 \mu \mathrm{m}$. Air is pressurized at the core with 1000 bar at 293k temperature. From the figure it can be observed that 1000 bar pressurized air-filled hollow core has greater confinement then the air-filled porous core at $\mathrm{f}=1 \mathrm{THz}$.

\section{Simulation AND ANALYSIS}

Finite element method (FEM) has been used throughout the simulation procedure with extra fine physic control mesh.FEM has been used to solve for Maxwell's equation. FEM is a numerical technique for finding approximate solution [26]; also referred as finite element analysis (FEA). FEM subdivides a large problem into smaller, simpler, parts, called finite elements. The simple equations that model these finite elements are then assembled into a larger system of equations that models the entire problem. FEM then uses variation methods from the calculus of variations to approximate a solution by minimizing an associated error function. A perfectly matched layer (PML) is imposed on the outside of the cladding region as it is the most efficient absorption condition. It improves calculation mode propagation in optical fiber of the proposed design.

For the calculation of more accurate effective index and to determine the accurate refractive index of air, Sellmeier equation of two terms and four parameters have been used [28]. The expression of Sellmeier equation is as follows-

$$
n^{2}(\lambda, p, T)=1+\frac{p}{p_{0}} \frac{T_{0}}{T}\left[\frac{B_{1} \lambda^{2}}{\lambda^{2}-C_{1}}+\frac{B_{2} \lambda^{2}}{\lambda^{2}-C_{2}}\right]
$$

All the coefficients termed as B1, B2, C1, C2 are found in table II of [28]. The other parameter termed as operating wavelength, $P_{0}$ is the pressure in bar and $T$ is temperature, 273 $\mathrm{K}$. For calculation of the EML or material absorption loss, $\alpha_{\mathrm{eff}}$ the following expression is used [11]

$$
\alpha_{\text {eff }}=\frac{\alpha_{\text {mode }}}{\alpha_{\text {mat }}}=\frac{\sqrt{\left(\frac{\varepsilon_{0}}{\mu_{0}}\right)} \int_{A_{\text {mat }}} \eta \alpha_{\text {mat }}|E|^{2} d A}{2 \int_{A l l} S_{2} d A}=\alpha_{\text {mat }} \eta \ldots
$$

In (2), $\varepsilon_{0}=$ permittivity of the vacuum, $\mu_{0}=$ permeability of the vacuum, $\eta=$ absorption loss ratio, $\alpha_{\mathrm{mat}}=$ material absorption loss, $S_{\mathrm{z}}=$ pointing vector in the direction of propagation $=1 / 2 \times \operatorname{Re}\left(E \times H^{*}\right) \mathrm{z}, \mathrm{E}=$ Electric field component and $\mathrm{H}=$ Magnetic field component. The integrator of the numerator is performed over the solid material region of TOPAS polymer. Here the designed kagome PCF is designed in only TOPAS material. For this reason, the confinement loss exists only in the PCF. Confinement indicates the light restricting ability of the core of the PCF. For calculation of the confinement loss the following expression is used [27].

$$
\alpha_{C}=\left(\frac{4 \pi f}{c}\right) I_{m}\left(\eta_{e f f}\right) \mathrm{cm}^{-1}=\left(4.187 \times 10^{4}\right) I_{m}\left(\eta_{\text {eff }}\right) \mathrm{cm}^{-1}
$$

In equation (3), $f=$ frequency of light which is constant for proposed design $=1 \mathrm{THz}, c=$ speed of light in vacuum i.e. $3 \times 10^{8} \mathrm{~ms}^{-1}$ and, $I_{\mathrm{m}}\left(\eta_{\text {eff }}\right)$ is the imaginary part of the effective index of the guided mode for the proposed kagome PCF.
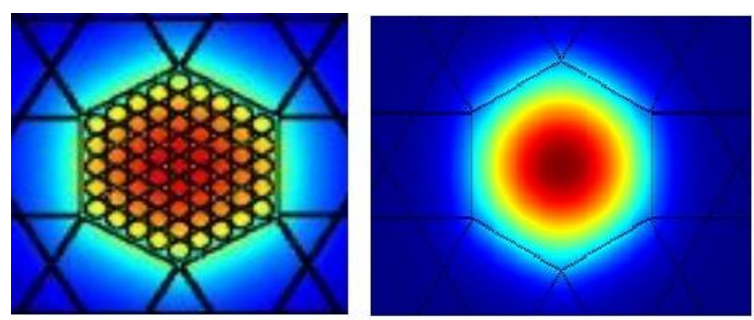

Fig. 5. Mode field confinement of both structure of porous core (left side) and $\mathrm{HC}-\mathrm{PCF}$ (right side) for frequency of $1 \mathrm{THz}$

Fig.5 shows the mode field confinement of both the proposed structure of $\mathrm{HC}-\mathrm{PCF}$ and porous core PCF at fixed $1 \mathrm{THz}$ of operating frequency. With the designed parameter and the pressurized core for HC-PCF the confinement is better in $\mathrm{HC}-\mathrm{PCF}$ than porous core PCF. The material loss and confinement loss has been calculated using (2) and (3) with the coefficients from [28] for HC-PCF and the simulation has been plotted against the core parameter and analyzed below.

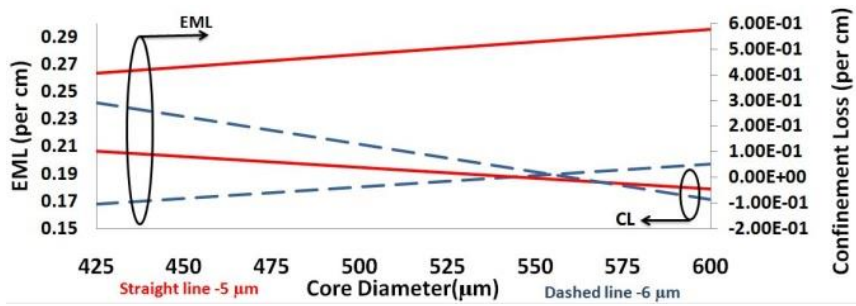

Fig. 6. EML and Confinement loss as a function of core diameter for various strut width and core porosity of $60 \%$ and $70 \%$ for $\mathrm{f}=1 \mathrm{THz}$ of porous core PCF.

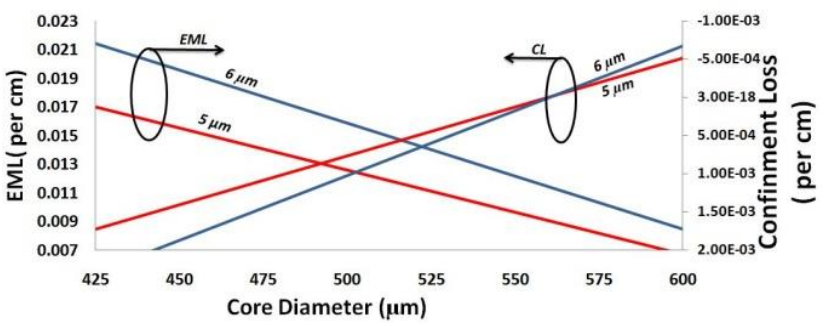

Fig. 7. EML and Confinement loss as a function of core diameter for various strut width and $\mathrm{f}=1 \mathrm{THz}$ of $\mathrm{HC}-\mathrm{PCF}$

EML and confinement loss of the designed porous core PCF has been simulated against various core diameters and plotted in fig.6. From the graph it is observed that the EML and confinement loss is inversely proportional to each other. While at $5 \mu \mathrm{m}$ of strut width the lowest EML recorded for $70 \%$ of core porosity and results as $0.166 \mathrm{~cm}-1$ at $1 \mathrm{THz}$ frequency. Again with further variation of strut width i.e. at $6 \mu \mathrm{m}$ the 
lowest EML calculated as $0.167 \mathrm{~cm}-1$. Generally there is not much difference in EML with variation in strut width. And in both cases they are showing lower loss than the bulk material absorption loss of TOPAS which is $0.2 \mathrm{~cm}-1$.

Again in fig.7, EML and confinement loss of the proposed structure of HC-PCF has been plotted to compare with porous core PCF. The same relationship recorded in fig.6is has been observed while conducting simulation for $\mathrm{HC}-\mathrm{PCF}$. The lowest EML recorded as $0.007 \mathrm{~cm}$ - 1 for core diameter of 600 $\mu \mathrm{m}$ at strut width of $5 \mu \mathrm{m}$ for $\mathrm{f}=1 \mathrm{THz}$. Again with increased strut width i.e. for $\mathrm{d}=6 \mathrm{um}$, the EML increased by $22 \%$. So lowest strut width would result low material absorption loss. Variation of core diameter results similar relationship; whereas at $425 \mu \mathrm{m}$ of core diameter shows $61 \%$ more EML than calculated for $600 \mu \mathrm{m}$. The calculated ratio remains same while variance occurs at $6 \mu \mathrm{m}$ of strut width.

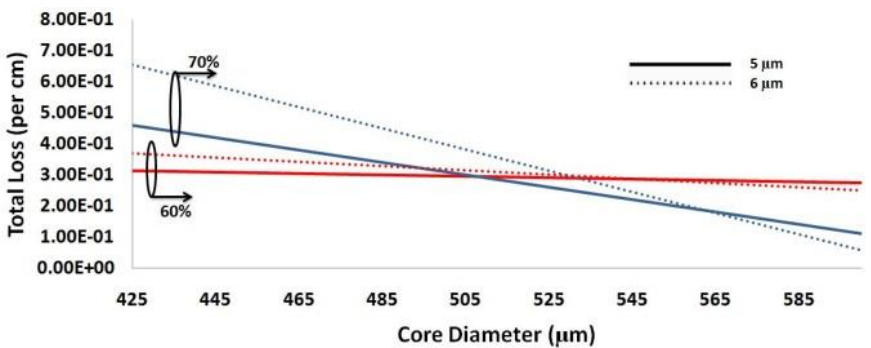

Fig. 8. Total loss as a function of core diameter of core porosity of $60 \%$ and $70 \%$ for various strut width at $\mathrm{f}=1 \mathrm{THz}$ for porous core $\mathrm{PCF}$

Fig. 8 indicates the total loss against the core diameter for the proposed structure of porous core at $1 \mathrm{THz}$; whereas total loss consists of both EML and confinement loss. With different core porosity the loss varies accordance with the core diameter. At $5 \mu \mathrm{m}$ the loss calculated is the lowest for any core porosity. For the performed simulation the lowest total loss recorded is $0.0197 \mathrm{~cm}-1$ at $600 \mu \mathrm{m}$ of core diameter and $70 \%$ of core porosity which varies approx. $32 \%$ more of the loss simulated at $60 \%$ core porosity. Hence, for proposed structure, core porosity is proportional to the total loss.

TABLE I. COMPARATIVE ANALYSIS ON HC-PCF AND POROUS CORE PCF

\section{Frequency $=1 \mathrm{THz}$}

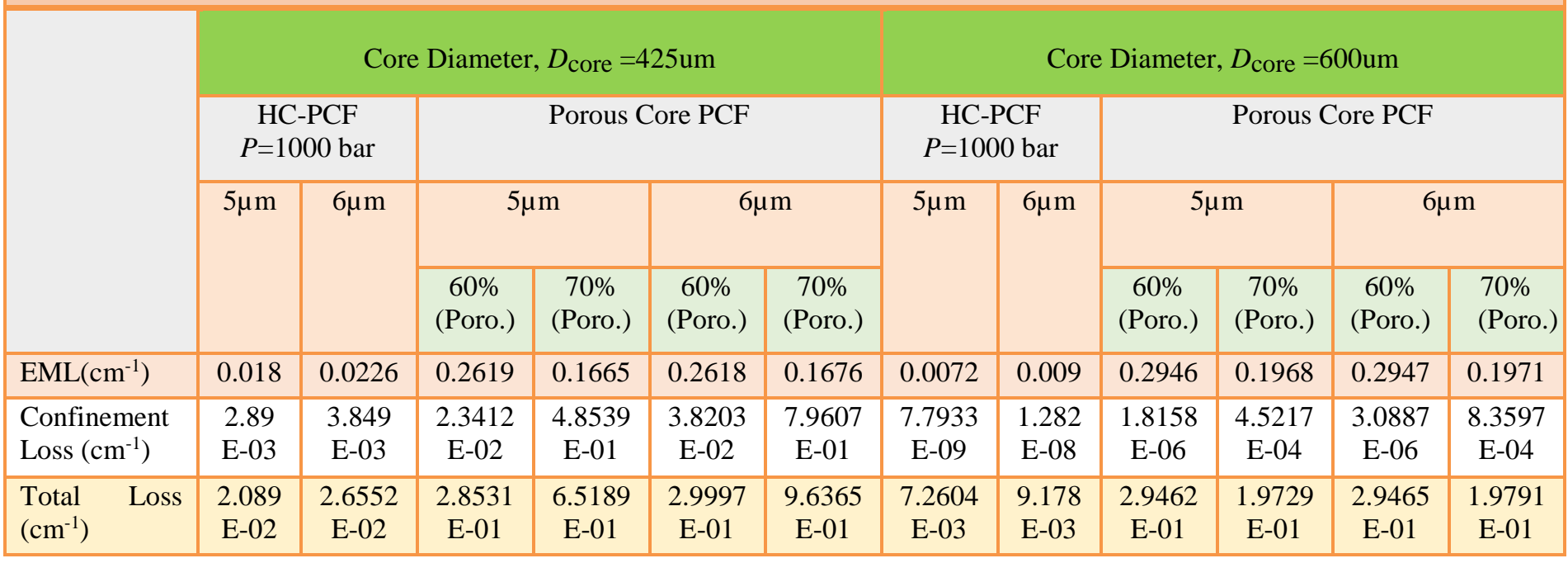

\section{RESUlt AND DiscuSSION}

Table 1 is showing the comparative analysis between the HC-PCF, and the porous core PCF. In the table 1, EML, confinement loss and total loss have been showed for both the proposed PCF structure at $\mathrm{f}=1 \mathrm{THz}$ and for various parameter. From the table 1, it can be observed that HC-PCF has lower loss recorded than porous core PCF.

The total loss calculated against the core diameter has been plotted in fig. 9 for comparing the result and choose a better wave guiding mechanism, for proposed HC-PCF. Core diameter is inversely proportional to the total loss. The lowest loss calculated is $0.00726 \mathrm{~cm}-1$ for $\mathrm{d}=5 \mu \mathrm{m}$, Dcore $=600$ $\mu \mathrm{m}$, and $\mathrm{f}=1 \mathrm{THz}$. With strut width variation from $5 \mu \mathrm{m}$ to 6 $\mu \mathrm{m}$ the loss increased up to $20 \%$. Again, by varying the core diameter the total loss show $65 \%$ increment from $425 \mu \mathrm{m}$ to $600 \mu \mathrm{m}$. Hence, the lowest loss can be found at higher core diameter and with optimized lowest strut width possible.

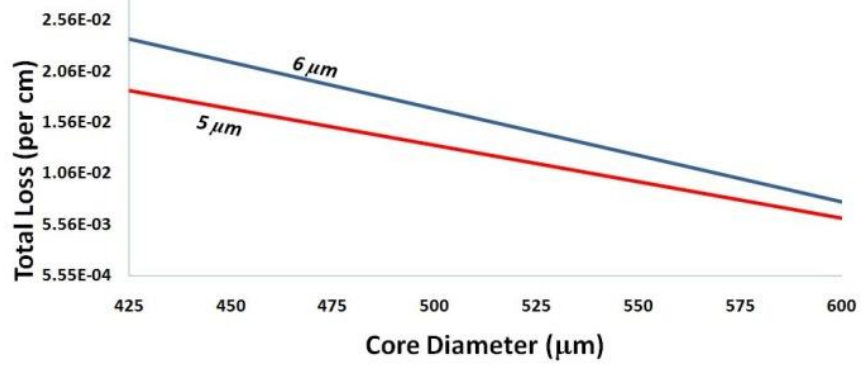

Fig. 9. Total loss as a function of core diameter for various strut widths at 1 $\mathrm{THz}$ of $\mathrm{HC}-\mathrm{PCF}$

By comparing the above simulation results, a comparative analysis graph has been plotted against the core diameter at $1 \mathrm{THz}$ of frequency in fig.10. Such comparison with the core porosity variation, strut width variation, and core diameter variation to calculate an optimized solution of EML, 
confinement loss and hence, total loss; proposed a solution for low material loss wave guidance system.

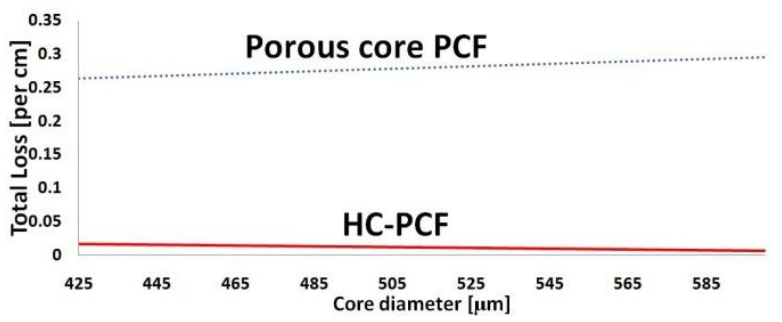

Fig. 10. A comparative analysis of Total loss as a function of core diameter for $\mathrm{f}=1 \mathrm{THz}$

For this work, HC-PCF is the optimized solution where the lowest loss calculated is $0.007 \mathrm{~cm}^{-1}$ which is approx. $95 \%$ lowest EML recorded than the porous core PCF reported over the current design. The confinement loss is also much lower than reported.

\section{Conclusion}

In conclusion, a comparative analysis is presented here for an optimized solution of low EML and confinement loss. The lowest loss recorded is $0.007 \mathrm{~cm}-1$ for $\mathrm{HC}-\mathrm{PCF}$ and found atf $=1 \mathrm{THz}$, Dcore $=600 \mu \mathrm{m}$ and $\mathrm{d}=5 \mu \mathrm{m}$ and pressurized air at 1000 bar. Comparatively lowest strut width and higher core diameter can results extremely low EML for proposed design. Such variance results approx. 95\% of improvement over the reported design with hollow core and pressure at 1000 bar. Increased pressure, can also results improvement over the current reported design. In comparison, to find for a better option between hollow core and porous core with air core inside and pressurized at 1000 bar for $\mathrm{HC}-\mathrm{PCF}$, hollow core kagome structured $\mathrm{THz}$ waveguide is better solution than porous core PCF. Simulation results are shown to asses this optimization and hence compared for improved solution. The findings can offer a significant improvement in biomedical $\mathrm{THz}$ waveguide designing and the scope for better improvement is always possible

\section{REFERENCES}

[1] Terahertz optics taking off [Editorial]. (2013, August 29). Nature Photonics. Retrieved November 5, 2016 from http://www.nature.com/nphoton/journal/v7/n9/full/nphoton.2013.239.ht $\mathrm{ml}$

[2] Sypek, Maciej. "From visible to THz optics" Photonics Letters of Poland vol. 4, no.3 pp-87, September 2012.

[3] C.J. Strachan, P.F. Taday, D.A. Newnham, K.C. Gordon, J.A. Zeitler, M. Pepper, T. Rades, "Using terahertz pulsed spectroscopy to quantify pharmaceutical polymorphism and crystallinity”,J. Pharm. Sci. vol. 94 pp. 837-846, April, 2005.

[4] M. M. Awad and R. A. Cheville, "Transmission terahertz waveguide based imaging below the diffraction limit," Appl. Phys. Lett., vol. 86, no. 22, pp. 221107-1-221107-3, May 2005.

[5] D.J. Cook, B.K. Decker, M.G. Allen, "Quantitative THz spectroscopy of explosive materials" Proc. OSA Conf., Opt. Terahertz Sci. Technol., pp. 1-4, April 2008.

[6] R.M. Woodward, V.P. Wallace, D.D. Arnone, E.H. Linfield, M. Pepper, "Terahertz pulsed imaging of skin cancer in the time and frequency domain", J. Biol. Phys.vol. 29 no. 2/3pp. 257-261, June 2003

[7] D.A. Crawley, C. Longbottom, B.E. Cole, C.M. Ciesla, D. Arnone, V.P. Wallace, M.Pepper, "Terahertz pulse imaging: a pilot study of potential applications in dentistry", Caries Res. vol. 37 pp. 352-359, September 2003.

[8] X.C. Zhang, and X. Jingzhou, "Introduction to $\mathrm{THz}$ wave photonics. New York: Springer, Vol. 29. January 2010
[9] R. Köhler,, A. Tredicucci,, F. Beltram,, H. E. Beere,, E. H. Linfield,, A. G. Davies, D.A. Ritchie, R. Clotti, \& F. Rossi, "Terahertz semiconductor-hetero structure laser". Nature, vol. 417, no. 6885 , pp. 156-159. March, 2002.

[10] Q. Wu, M. Litz, and X. C. Zhang, "Broadband detection capability of ZnTe electro-optic field detectors," Appl. Phys. Lett., vol. 68, no. 21, pp. 2924-2926, March 1996

[11] G. K. M. Hasanuzzaman, M. S. Habib, S. M. Abdur Razzak, M. A. Hossain, and Y. Namihira, "Low Loss Single-Mode Porous-Core Kagome Photonic Crystal Fiber for THz Wave Guidance," J. Lightwave Technol. Vol. 33, pp. 4027-4031, October, 2015

[12] H. Bao, K. Nielsen, H. K. Rasmussen, P. U. Jepsen, \& O. Bang, "Fabrication and characterization of porous-core honeycomb band gap THz fibers," Opt. Exp., vol. 20, no. 28, pp. 29507-29517, December, 2012

[13] K. Nielsen, H.K. Rasmussen, P.U. Jepsen, O. Bang, "Porous-core honeycomb band gap THz fiber,” Opt. Lett., vol. 36, no. 5, pp. 666-668, March, 2011.

[14] J.C. Knight, T.A. Birks, P. St. J. Russell, D M. Atkin "All-silica single mode optical fiber with photonic crystal cladding". Optics Lett., vol. 21, no. 19,pp. 1547-1549, October 1996.

[15] H. Han, H. Park, M. Cho, \&J. Kim,"Terahertz pulse propagation in a plastic photonic crystal fiber". Applied Physics Letters, vol. 80. No. 15, pp. 2634-2636, April 2002.

[16] S. Atakaramians, S. Afshar, T. M. Monro, \&D. Abbott, "Terahertz dielectric waveguides. Advances in Optics and Photonics", vol. 5, no. 2, pp. 169-215, February 2003

[17] P. St. J. Russel, "Photonic crystal fiber" et. al. Science pp. 299, pp. 358,May 2003.

[18] J.-Y. Lu, C.-P. Yu, H.-C. Chang, H.-W. Chen, Y.-T. Li, C.-L. Pan, and C.-K. Sun, "Terahertz air-core microstructure fiber," Appl. Phys. Lett. 92, no. 064105, February, 2008.

[19] O. Mitrofanov, J. Richards, F.A. Farnandez, T.K. Mavrogordatos, J.A. Harrington, "Reducing transmission losses in hollow THz waveguides." IEEE Transactions on Terahertz Science and Technology vol. 1, no. 1 pp. 124-132, 2011

[20] O. H. Heckl, C. R. E. Baer, C. Kränkel, S. V. Marchese, F. Schapper, M. Holler, \&P. Light, "High harmonic generation in a gas-filled hollowcore photonic crystal fiber". Applied Physics B, vol. 97 no. 2, pp. 369373, October 2009.

[21] C.J. Hensley, M.A. Foster, B. Shim, A.L. Gaeta, "Extremely high coupling and transmission of high-powered femto second pulses in hollow-core photonic band-gap fiber", in Conference on Lasers and Electro-Optics (CLEO) (IEEE Press, New York, 2008), p. paper JFG1, October 2009

[22] M. Azhar, G. K. L. Wong, W. Chang, N. Y. Joly, P. St. J. Russell, "High pressure gases in hollow core photonic crystal fiber: A new nonlinear medium". arXiv preprint arXiv:1210.3482, October, 2012.

[23] G. J. Pearce, G. S. Wieder hecker, C. G. Poulton, Burger, S., \&P. St. J. Russell, "Models for guidance in kagome-structured hollow-core photonic crystal fibers." Optics express, vol. 15, no. 20, pp. 1268012685, 2007.

[24] J. Liang, R. Liyong, "Broadband, low-loss, dispersion flattened porouscore photonic bandgap fiber for terahertz (THz)-wave propagation." Optics Communications vol. 295pp. 257-261, January 2013.

[25] M. Uthman, B. M. A. Rahman, N. Kejalakshmy, A. Agrawal, \&K. T. V. Grattan, "Design and characterization of low-loss porous-core photonic crystal fiber". IEEE Photonics Journal, vol. 4, no. 6, pp. 2315-2325, December 2012

[26] S. F. Kaijage, Z. Ouyang, and X. Jin, "Porous-core photonic crystal fiber for low loss terahertz wave guiding," IEEE Photon. Technol. Lett., vol. 25, no. 15, pp. 1454-1457, August, 2013.

[27] S.Ali,, N. Ahmed, S. Aljunid, and B. Ahmad. "Ultra-flat low materia loss porous core $\mathrm{THz}$ waveguide with near zero flat dispersion." Electronics Lett. vol. 52, no. 10, pp: 863-865, May, 2016.

[28] A. Börzsönyi, Z. Heiner, M. P. Kalashnikov, A. P. Kovács, and K. Osvay, "Dispersion measurement of inert gases and gas mixtures at 800 nm," Appl. optics vol. 47, no. 27, pp. 4856-4863, September 2008. 


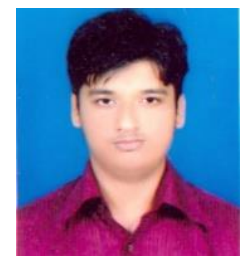

Mohaiminul Islam received B.Sc. Engg. (EEE) degree from American International UniversityBangladesh (AIUB) in 2015 and completed Masters in EEE (MEEE) from AIUB in 2017. $\mathrm{He}$ is a Lecturer in European University of Bangladesh. His research interests are on the field of photonics.

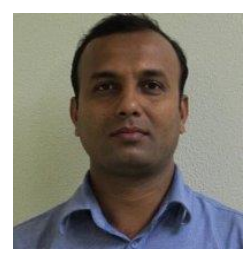

Md. Anwar Hossain received the B.Sc. Engg. (EEE) from the Rajshahi University of Engineering \& Technology, Bangladesh in 2001, the M.Sc. Engg. Degree in information and communication technology from The Asian Institute of Technology in2006 and the Ph.D. Engg. Degree in electronics and information

engineering under Interdisciplinary Intelligent Systems Engineering from the University of the Ryukus, Nishihara, Okinawa, Japan in 2013. He received Post-doctoral Fellowship of Japan Society for the Promotion of Science and he is currently working as an Associate Professor in department of EEE, American International UniversityBangladesh, Dhaka, Bangladesh

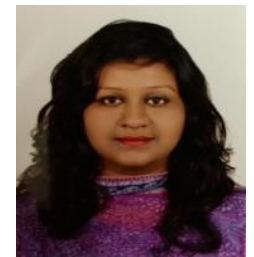

Fahmida Haque received B.Sc. Engg.(EEE) degree from American International UniversityBangladesh (AIUB) in 2015. She is a Lecturer in European University of Bangladesh. Her research interests are on the field of photonics. 